University of New Hampshire

University of New Hampshire Scholars' Repository

Space Science Center

Institute for the Study of Earth, Oceans, and

Space (EOS)

2008

\title{
POET: POlarimeters for Energetic Transients
}

J E. Hill

NASA Goddard Space Flight Ctr.

Mark L. McConnell

University of New Hampshire - Main Campus, mark.mcconnell@unh.edu

Peter F. Bloser

University of New Hampshire, Peter.Bloser@unh.edu

Jason S. Legere

University of New Hampshire, jslegere@unh.edu

John R. Macri

University of New Hampshire - Main Campus, John.Macri@unh.edu

See next page for additional authors

Follow this and additional works at: https://scholars.unh.edu/ssc

Part of the Astrophysics and Astronomy Commons

\section{Recommended Citation}

POET: POlarimeters for Energetic Transients Hill, J. E. and McConnell, M. L. and Bloser, P. and Legere, J. and Macri, J. and Ryan, J. and Barthelmy, S. and Angelini, L. and Sakamoto, T. and Black, J. K. and Hartmann, D. H. and Kaaret, P. and Zhang, B. and loka, K. and Nakamura, T. and Toma, K. and Yamazaki, R. and Wu, X., AIP Conference Proceedings, 1065, 331-337 (2008), DOI:http://dx.doi.org/10.1063/1.3027941

This Conference Proceeding is brought to you for free and open access by the Institute for the Study of Earth, Oceans, and Space (EOS) at University of New Hampshire Scholars' Repository. It has been accepted for inclusion in Space Science Center by an authorized administrator of University of New Hampshire Scholars' Repository. For more information, please contact Scholarly.Communication@unh.edu. 


\section{Authors}

J E. Hill, Mark L. McConnell, Peter F. Bloser, Jason S. Legere, John R. Macri, James M. Ryan, Scott Barthelmy, L Angelini, Takanori Sakamoto, J Kevin Black, H Hartmann, Philip Kaaret, Bing Zhang, Kunihito Ioka, Takashi Nakamura, Kenji Toma, Ryo Yamazaki, and X Wu 


\section{AIP $\mid$ Proceedings}

\section{POET: POlarimeters for Energetic Transients}

J. E. Hill, M. L. McConnell, P. Bloser, J. Legere, J. Macri, J. Ryan, S. Barthelmy, L. Angelini, T. Sakamoto, J. K. Black, D. H. Hartmann, P. Kaaret, B. Zhang, K. loka, T. Nakamura, K. Toma, R. Yamazaki, and X. Wu

Citation: AIP Conference Proceedings 1065, 331 (2008); doi: 10.1063/1.3027941

View online: http://dx.doi.org/10.1063/1.3027941

View Table of Contents: http://scitation.aip.org/content/aip/proceeding/aipcp/1065?ver=pdfcov

Published by the AIP Publishing

\section{Articles you may be interested in}

RapidlyResponding Optical Polarimetry of GRB afterglows with Hiroshima 1.5m Telescope and Oneshot Wide field Polarimeter

AIP Conf. Proc. 1279, 355 (2010); 10.1063/1.3509309

A BalloonBorne GammaRay Polarimeter for GammaRay Bursts

AIP Conf. Proc. 1133, 61 (2009); 10.1063/1.3155968

Relationship of solar flare accelerated particles to solar energetic particles (SEPs) observed in the interplanetary medium

AIP Conf. Proc. 781, 246 (2005); 10.1063/1.2032704

Development of a hard x-ray polarimeter for gamma-ray bursts

AIP Conf. Proc. 428, 889 (1998); 10.1063/1.55415

Angle distribution and time variation of gamma ray flux from solar and stellar winds, 1. Generation by flare energetic particles

AIP Conf. Proc. 410, 1178 (1997); 10.1063/1.54099 


\title{
POET: POlarimeters for Energetic Transients
}

\author{
J. E. Hill*, M. L. McConnell ${ }^{\dagger}$, P. Bloser ${ }^{\dagger}$, J. Legere $^{\dagger}$, J. Macri ${ }^{\dagger}$, J. Ryan ${ }^{\dagger}$, S. \\ Barthelmy**, L. Angelini**, T. Sakamoto $^{* *}$, J. K. Black ${ }^{\ddagger}$, D. H. Hartmann ${ }^{\S}$, P. \\ Kaaret $^{\mathbb{T}}$, B. Zhang $\|$, K. Ioka ${ }^{\dagger \dagger}$, T. Nakamura ${ }^{\dagger \dagger}$, K. Toma ${ }^{\dagger \dagger}$, R. Yamazaki ${ }^{+\ddagger}$ and X. \\ $\mathrm{Wu}^{\S \S}$ \\ ${ }^{*}$ Universities Space Research Association, CRESST and NASA Goddard Space Flight Center, Greenbelt, MD \\ 20771 USA \\ ${ }^{\dagger}$ Space Science Center, University of New Hampshire, Durham, NH 03824, USA \\ ${ }^{* *}$ NASA Goddard Space Flight Center, Greenbelt, MD 20771, USA \\ ${ }^{\ddagger}$ Rock Creek Scientific, 1400 East-West Hwy, Suite 807, Silver Spring, MD 20910, USA \\ ${ }^{\S}$ Department of Physics and Astronomy, Clemson University, Clemson, SC 29634 \\ I Department of Physics and Astronomy, University of Iowa, Iowa City, IA 52242, USA \\ $\|$ Department of Physics, University of Nevada, Box 454002, Las Vegas, NV 89154, USA \\ ${ }^{\dagger}$ Theoretical Astrophysics Group, Department of Physics, Kyoto University, Sakyo-ku, Kyoto 606-8502, Japan \\ ${ }^{*}$ Department of Physical Science, Hiroshima University, Higashi-Hiroshima, 739-8526, Japan \\ ${ }^{\$}$ Purple Mountain Observatory, Chinese Academy of Sciences, Nanjing 210008, China
}

\begin{abstract}
POET (Polarimeters for Energetic Transients) is a Small Explorer mission concept proposed to NASA in January 2008. The principal scientific goal of POET is to measure GRB polarization between 2 and $500 \mathrm{keV}$. The payload consists of two wide FoV instruments: a Low Energy Polarimeter (LEP) capable of polarization measurements in the energy range from 2-15 keV and a high energy polarimeter (Gamma-Ray Polarimeter Experiment - GRAPE) that will measure polarization in the $60-500 \mathrm{keV}$ energy range. Spectra will be measured from $2 \mathrm{keV}$ up to $1 \mathrm{MeV}$. The POET spacecraft provides a zenith-pointed platform for maximizing the exposure to deep space. Spacecraft rotation will provide a means of effectively dealing with systematics in the polarization response. POET will provide sufficient sensitivity and sky coverage to measure statistically significant polarization for up to $100 \mathrm{GRBs}$ in a two-year mission. Polarization data will also be obtained for solar flares, pulsars and other sources of astronomical interest.
\end{abstract}

Keywords: Gamma-ray Bursts, Polarimetry, X-ray, Gamma-ray

PACS: $95.55 . \mathrm{Ka}$

\section{INTRODUCTION}

Gamma-ray bursts (GRBs) are the most explosive events in the universe, and have stimulated intense observational and theoretical research. Theoretical models indicate that an understanding of the inner structure of GRBs, including the geometry and physical processes close to the central engine, requires the exploitation of high energy X-ray and gammaray polarimetry but observational techniques have been limited. Recent advances in instrument capabilities finally enable the exploration of polarization of X-ray and gamma-ray emissions from GRBs. POlarimeters for Energetic Transients (POET) is a SMEX mission concept that provides highly sensitive polarimetric observations of GRBs and can also make polarimetry measurements of solar flares, pulsars, soft gamma repeaters, and slow transients. POET will make measurements with two different polarimeters (both with wide fields of view) to provide a broad energy range of observations: The Gamma-RAy Polarimeter Experiment (GRAPE; 60-500 kev) and the Low Energy Polarimeter (LEP; $2-15 \mathrm{keV}$ ). The POET mission would significantly advance our understanding of key physical processes through high energy polarimetry.

\section{GAMMA-RAY BURST SCIENCE WITH POET}

Extensive multi-wavelength observations of the prompt GRB emission and the long-wavelength afterglows have led to the development of models for two types of GRBs [21]. Long bursts ( $>2 \mathrm{~s}$ ) typically exhibit a relatively soft spectrum 
and are generally associated with the death of massive stars[12] whereas short bursts $(<2 s)$ generally exhibit a harder spectrum and are associated with the merger of compact star binaries (e.g., neutron star-neutron star, neutron starblack hole, etc., [4]. In both cases, the process is thought to result in the formation of a black hole. Regardless of the progenitor and the central engine, a generic "fireball" model suggests that a relativistic jet is launched from the center of the explosion, with a bulk Lorentz factor, $\Gamma$, greater than 100 [13]. The "internal" dissipation within the fireball (likely due to internal shocks) leads to emission in the X-ray and gamma-ray band, which corresponds to the observed GRB prompt emission. Eventually, the fireball jet is decelerated by the circumburst medium, which leads to a longlasting forward shock, the emission from which is believed to be responsible for the long-lasting afterglows following the bursts themselves.

In spite of extensive observational efforts (e.g., CGRO, HETE-2, BeppoSAX, INTEGRAL, Swift, and others), several key properties of GRB explosions remain poorly understood and are difficult or even impossible to infer with the spectral and lightcurve information currently collected. High energy polarization measurements will lead to unambiguous answers to many open questions, including:

What is the magnetic structure of GRB jets? It is speculated that strong magnetic fields are generated at the GRB central engine, which may play an essential role in the launch of the relativistic jets. It is unclear, however, whether the GRB emission region is penetrated by a globally structured, dynamically important magnetic field, and whether GRB emission is due to shock dissipation or magnetic reconnection.

What is the geometric structure of GRB jets? Although it is generally believed that GRBs are collimated, the distribution of jet opening angles and the observer's viewing direction are not known, and it is not clear whether there are small-scale structures within the global jet.

What is the prompt radiation mechanism of GRBs? The leading model is synchrotron emission of relativistic electrons in a globally ordered magnetic field carried from the central engine or random magnetic fields generated in situ in the shock dissipation region. Other suggestions include Compton drag of ambient soft photons, synchrotron self-Compton emission, and the combination of a thermal component from the photosphere and a non-thermal component (e.g., synchrotron).

The theoretical models for the prompt emission generally fall into two broad types [20]: The physical model invokes a globally ordered magnetic field in the emission region, so that electron synchrotron emission in this ordered field gives a net linear polarization (e.g., [20][8][5]). Such a model applies for most observer viewing-angle geometries, where the typical level of polarization is quite high ( $\Pi>20 \%$ ), with the maximum $\Pi \sim 70 \%$. The geometric model requires an optimistic viewing direction to observe a high degree of polarization. In this model, both the magnetic field structure and electron energy distribution is random in the emission region so that no net polarization is detected if the viewing angle is along the jet beam (regardless of the radiation mechanism). However, if the viewing direction is near the edge of the jet, in particular about $1 / \Gamma$ outside the jet cone, a high polarization degree would result due to loss of the emission symmetry [17][20][7]. Within the context of the geometric model, typical polarization values are $\Pi<20 \%$, although synchrotron emission can produce as high as $\Pi \sim 70 \%$ and Compton drag models [7] can achieve $\Pi \sim 100 \%$ under optimistic geometric configurations.

In general, given a random distribution of viewing angles, the fraction of bursts that can achieve a high $\Pi$ in the geometric models is significantly smaller than that in the physical models. A statistical study of polarization properties of a large sample of GRBs would therefore differentiate between the models, and provide a direct diagnostic of the magnetic field structure, radiation mechanism and geometric configuration of GRB jets. Toma et al. [19] displays the predictions for the distribution of polarization magnitudes and the dependence of $\Pi$ on the peak of the energy break $\left(E_{p}\right)$ in the GRB spectrum. Results are shown for three distinct models:

- Physical model for synchrotron emission with ordered magnetic fields (SO)

- Geometric model for synchrotron emission in random magnetic fields (SR)

- Geometric model for Compton-drag (CD)

The POET sensitivity will allow the accumulation of GRB polarization measurements at a rate of $\sim 50$ /year, permitting studies that will distinguish between the geometric and physical models. Given a sufficiently large number of events, it may even be possible to distinguish between the two geometric models (SR and CD).

The GRB radiation mechanism can be determined from the energy-dependence of the polarization measurements. The GRB prompt emission spectrum is typically characterized by a broken powerlaw [1]. Generally it is believed that spectral energy break $\left(E_{p}\right)$ corresponds to a break in the non-thermal electron spectral distribution. This would result in a jump of the polarization degree across $E_{p}$. Alternatively, some authors (e.g., [15]) argue that the observed spectrum is 
TABLE 1. Instrument Parameters

\begin{tabular}{lll}
\hline & GRAPE & LEP \\
\hline Polarimetry & $60-500 \mathrm{keV}$ & $2-15 \mathrm{keV}$ \\
Detectors & BGO/plastic scintillator (62) & $\mathrm{Ne}: \mathrm{CO}_{2}: \mathrm{CH}_{3} \mathrm{NO}_{2} \mathrm{Gas}(8)$ \\
Spectroscopy & $15 \mathrm{keV}-1 \mathrm{MeV}$ & $2-15 \mathrm{keV}$ \\
Detectors & $\mathrm{NaI}(\mathrm{TI})$ scintillator (2) & as above \\
Field-of-View & $\pm 60^{\circ}$ & $\pm 44^{\circ}$ \\
\hline
\end{tabular}

a superposition of a thermal spectrum (probably from the photosphere) and a non-thermal synchrotron spectrum. This would lead to a dip in the polarization spectrum. POET can measure the degree of polarization both above and below $E_{p}$ (or measure the polarization as a function of energy) and therefore would differentiate the two models, and identify the GRB radiation mechanism and the emission site, which are very difficult to infer from current observations.

\section{POET INSTRUMENT SUITE}

POET is comprised of two polarimetry instruments, GRAPE and LEP, co-aligned on a zenith pointed rotating spacecraft. LEP and GRAPE determine polarization by measuring the number of events versus the event azimuth angle (EAA) as projected onto the sky. This is referred to as a modulation profile and represents a measure of the polarization magnitude and direction of polarization for the incident beam. Depending on the type of polarimeter, the EAA is either the direction of the ejected photoelectron (LEP) or the direction of the scattered photon (GRAPE). The response of a polarimeter to $100 \%$ polarized photons can be quantified in terms of the modulation factor, $\mu$, which is given by:

$$
\mu=\frac{C_{\max }-C_{\min }}{C_{\max }+C_{\min }}
$$

Where $C_{\max }$ and $C_{\min }$ are the maximum and minimum of the modulation profile, respectively. The polarization fraction ( $\Pi$ ) of the incident flux is obtained by dividing the measured modulation by that expected for $100 \%$ polarized flux. The polarization angle $\left(\phi_{o}\right)$ corresponds either to the maximum of the modulation profile (LEP) or the minimum of the modulation profile (GRAPE). To extract these parameters from the data, the modulation histograms are fit to the functional form:

$$
C(\Phi)=A+B \cos ^{2}\left(\Phi-\Phi_{o}\right)
$$

The sensitivity of a polarimeter is defined in terms of the MDP, which refers to the minimum level of polarization that is detectable with a given observation (or, equivalently, the apparent polarization arising from statistical fluctuations in unpolarized data). The precise value of the MDP will depend on the source parameters (fluence, spectrum, etc.) and the polarimeter characteristics. At the 99\% confidence level, the MDP can be expressed as,

$$
M D P=\frac{4.29}{\varepsilon \mu}\left(\frac{\varepsilon S A+B}{t}\right)^{1 / 2}
$$

where $\mathrm{S}$ is the source strength (cts $\mathrm{cm}^{-2} \mathrm{~s}^{-1}$ ), B is the total background rate (cts $\mathrm{cm}^{-2} \mathrm{~s}^{-1}$ ), $\mathrm{t}$ is the observing time (sec), $\varepsilon$ is the quantum efficiency, and $A$ is the collecting area. The ultimate sensitivity, however, may not be limited by statistics but by systematic errors created by false modulations that arise from azimuthal asymmetries in the instrument.

\section{Gamma-RAy Polarimeter Experiment: GRAPE}

GRAPE [9][10] is designed to measure polarization from $60-500 \mathrm{keV}$ and to provide spectroscopy over a broad energy range from $15 \mathrm{keV}$ to $1 \mathrm{MeV}$. The GRAPE design is highly modular and fault tolerant. The GRAPE instrument is composed of 64 independent detector modules arranged in two identical assemblies that provide the associated electronics and the required mechanical and thermal support. All detector modules employ well-established, hightechnology readiness level scintillator/photomultiplier tube (PMT) technology with geometries optimized by both simulation and laboratory studies. 

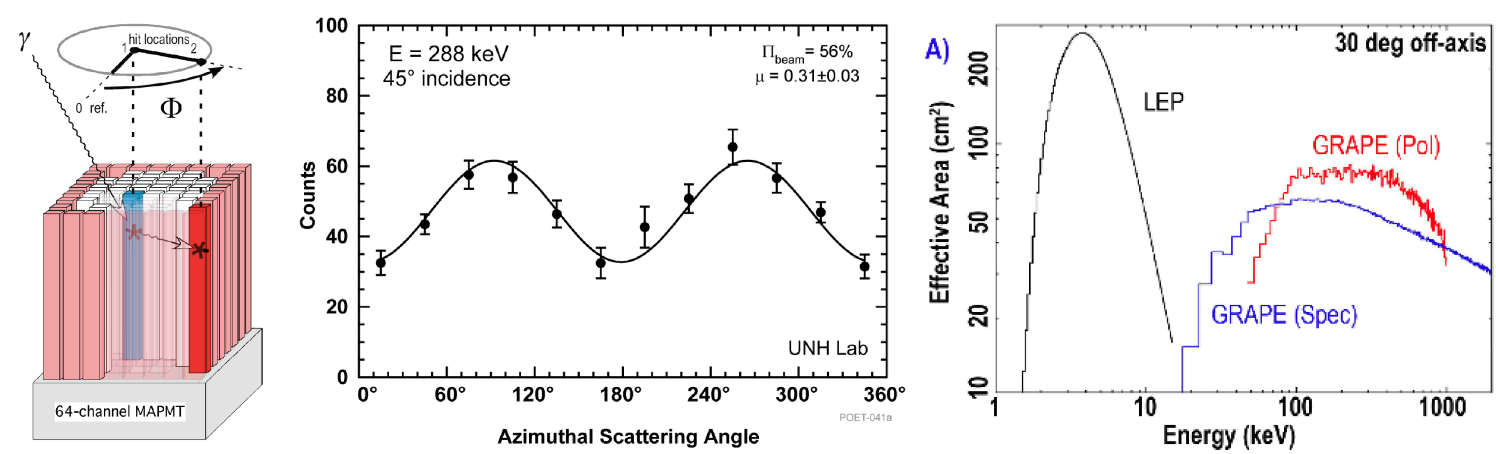

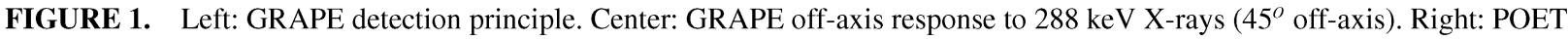
Effective area $30^{\circ}$ off-axis

At energies from $\sim 50 \mathrm{keV}$ up to several $\mathrm{MeV}$, photon interactions are dominated by Compton scattering. The operational concept for GRAPE is based on the fact that, in Compton scattering, photons are preferrentially scattered at a right angle to the incident electric field vector (the polarization vector). If the incident beam of photons is polarized, the azimuthal distribution of scattered photons will be asymmetric. The direction of the polarization vector is defined by the minimum of the scatter angle distribution. The GRAPE performance characteristics are shown in Table 1.

Polarimeter Modules: The design of the GRAPE instrument is very modular, with 62 independent polarimeter modules and 2 spectroscopy modules. Each polarimeter module incorporates an array of optically independent $5 \times 5 \times 50$ $\mathrm{mm}^{3}$ non-hygroscopic scintillator elements aligned with and optically coupled to the $8 \times 8$ scintillation light sensors of a 64-channel MAPMT. Two types of scintillators are employed. Low-Z plastic scintillator is used as an effective medium for Compton scattering. High-Z inorganic scintillator (Bismuth Germanate, BGO) is used as a calorimeter, for absorbing the full energy of the scattered photon. The arrangement of scintillator elements within a module has 28 BGO calorimeter elements surrounding 32 plastic scintillator scattering elements. Valid polarimeter events are those in which a photon Compton scatters in one of the plastic elements and is subsequently absorbed in one of the BGO elements, as shown in Figure 1. These events can be identified as a coincident detection between one plastic scintillator element and one BGO calorimeter element. The azimuthal scatter angle is determined for each valid event by the relative locations of hit scintillator elements. It is not necessary to know where within each element the interaction takes place (e.g., the depth of interaction). It is sufficient to know only the lateral location of each element to generate a histogram of photon scatter angles. Each independent polarimeter module includes the electronics required to process the MAPMT signals as well as qualify and digitize the event data. This approach - compact, rugged, independent modules - was employed successfully with two H8500 MAPMT-based detector modules in the GRAPE balloon payload flown from Palestine, TX in June 2007.

Spectrometer Modules: To facilitate spectral measurements over a broader energy range ( $15 \mathrm{keV}-1 \mathrm{MeV})$, GRAPE includes 2 spectrometer modules. Each spectrometer module assembly has a single thallium-doped sodium iodide $(\mathrm{NaI}(\mathrm{Tl}))$ scintillator crystal mounted on the face of the MAPMT. Laboratory tests of this configuration demonstrate energy resolution of $15 \%$ and $8 \%$ FWHM at 122 and $662 \mathrm{keV}$, respectively.

\section{Performance}

The scientific performance of the GRAPE polarimetry modules has been investigated using a laboratory prototype module and Monte Carlo simulations. The simulations were performed using the software package MGEANT [18] with a modification to include the effects of polarization in Compton scattering [10]. The simulations were validated with laboratory tests and have been used to predict the performance of the GRAPE instrument on POET.

Laboratory Testing: The laboratory prototype is a configuration of plastic scintillators and CsI crystals. The prototype module was exposed to partially polarized radiation produced by $90^{\circ}$ scattering of $662 \mathrm{keV}$ photons (from a ${ }^{137} \mathrm{Cs}$ source) in a plastic scintillator block, producing a beam with energy $288 \mathrm{keV}$ and a polarization of $\sim 60 \%$. The measured polarization for normally-incident radiation was $56 \% \pm 9 \%$ was consistent with the scattering geometry. Tests were also performed with an off-axis source to demonstrate the wide angle response of the GRAPE design at angles $>45^{\circ}$. The response is shown in Figure 1.

Beam Calibration: The prototype module was exposed to $100 \%$ polarized radiation of two energies at the Advance Photon Source at Argonne National Laboratory. Modulation factors of 46\% (69 keV) and 48\% (129 keV), were 

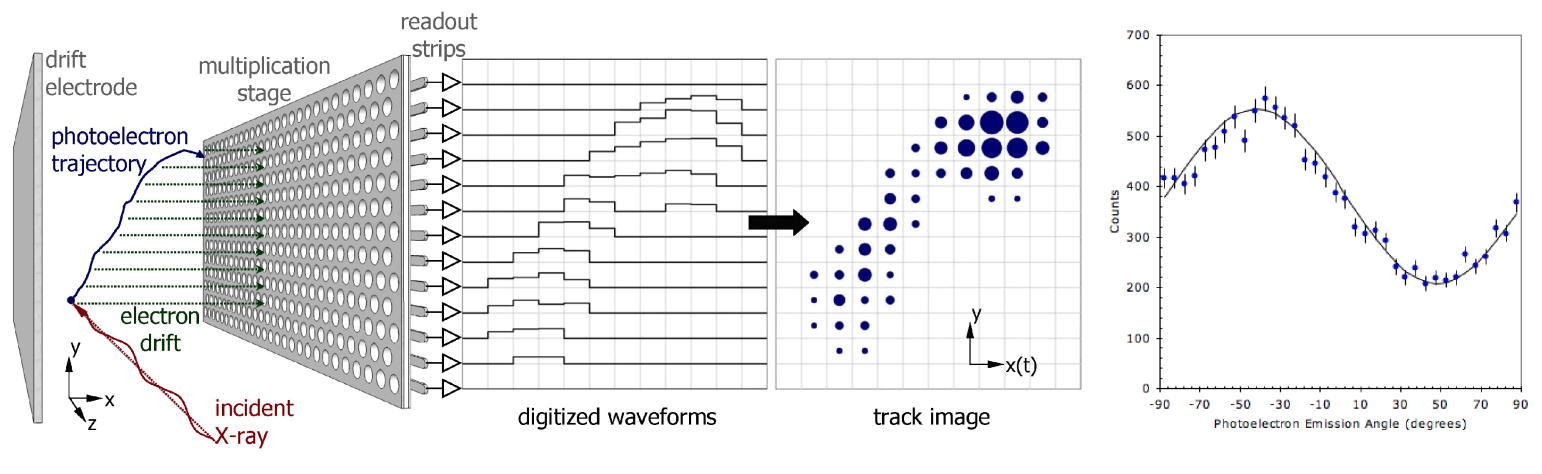

FIGURE 2. Left: The TPC polarimeter uses a simple strip readout and time of arrival to form a pixelized image of photoelectron track. Middle: The TPC polarimeter forms an image by digitizing the signal on each readout strip. The signal from a $6 \mathrm{keV}$ X-ray, proportional to the charge pulse-train deposited on each strip, is shown on the right. The resulting image shows the interaction point, emission angle and end of the track. The size of each circle is proportional to the deposited charge in each virtual pixel; the grid is on a $132 \mu \mathrm{m}$ spacing. Right: A histogram of reconstructed polarized $6.4 \mathrm{keV}$ photoelectron tracks. Demonstrates a modulation of $45 \%$.

obtained which are in agreement with simulations [10].

Engineering Balloon Flight: In June 2007 the prototype polarimeter module was flown on a NASA balloon flight from Palestine, TX. The module performance met all requirements throughout the flight. The measured background counting rate (integrated over the full energy range of the instrument) is $\sim 2 \mathrm{cts} \mathrm{sec}^{-1}$ per module.

Simulations: The full GRAPE instrument has been simulated to predict its scientific performance. Both the effective area and modulation factor were calculated using simulations of mono-energetic photons with $100 \%$ polarization. The off-axis effective area is shown as a function of energy in Figure 1. Simulations show that GRAPE retains $\sim 40 \%$ of its polarization sensitivity for photons incident $60^{\circ}$ off-axis. Based on these performance simulations, we expect GRAPE to detect $\sim 40$ GRBs per year with a MDP better than 20\%, and 6 per year with MDP better than $8 \%$.

\section{Low Energy Polarimeter: LEP}

The LEP measures the polarization of incident photons with the innovative operation of a Time Projection Chamber (TPC), a proven technology used in high-energy particle physics. It is simple in design and uses the photoelectric effect to provide unmatched broadband polarization sensitivity over the $2-15 \mathrm{keV}$ band-pass making it a low risk solution that requires low power and mass to make highly sensitive measurements [2][6].

TPC Polarimeter Operation: The LEP polarimeter enclosure will consist of four dual-readout detector modules each with an isolated gas volume contained by a Be X-ray window. Each detector module contains two 6 x 12 x 24 $\mathrm{cm}^{3}(\mathrm{LxWxH})$ TPCs that share a single X-ray transparent drift electrode. Each TPC is comprised of a micropattern proportional counter, consisting of a shared drift electrode and a high-field gas electron multiplier (GEM) positioned $1 \mathrm{~mm}$ from a strip readout plane. When an X-ray is absorbed in the gas between the drift electrode and the GEM, a photoelectron is ejected in a preferential direction with a $\cos ^{2} \phi$ distribution, where $\phi$ is the azimuthal angle measured from the X-ray polarization vector. As the photoelectron travels through the gas it creates a path of ionization that drifts in a moderate, uniform field to the GEM where an avalanche occurs. The charge finally drifts to the strip detector where it is read out.

Figure 2 illustrates how a track image projected onto the $x-y$ plane is formed by digitizing the charge pulse waveforms and binning into pixels. The coordinates are defined by strip location in one dimension, and arrival time multiplied by the drift velocity in the orthogonal dimension. The strips are smaller than the mean free path of the photoelectron and therefore an image of the track can be reconstructed and the initial direction of the photoelectron determined. The magnitude and orientation of the source polarization can be determined from a histogram of the emission angles.

Design: The LEP will use $12 \times 24 \mathrm{~cm}^{2}$ GEMs with holes in a hexagonal configuration on an $80 \mu \mathrm{m}$ pitch. The readout plane is $12 \times 24 \mathrm{~cm}^{2}$, with strip electrodes $24 \mathrm{~cm}$ long on a $80 \mu \mathrm{m}$ pitch and every $31^{\text {st }}$ strip tied together. The LEP will use nitromethane $\left(\mathrm{CH}_{3} \mathrm{NO}_{2}\right)$ as the charge-carrying ion in neon, with a small quantity of $\mathrm{CO}_{2}$ as a quench gas at 780 Torr providing a slower drift speed compatible with slower, low power electronics. 

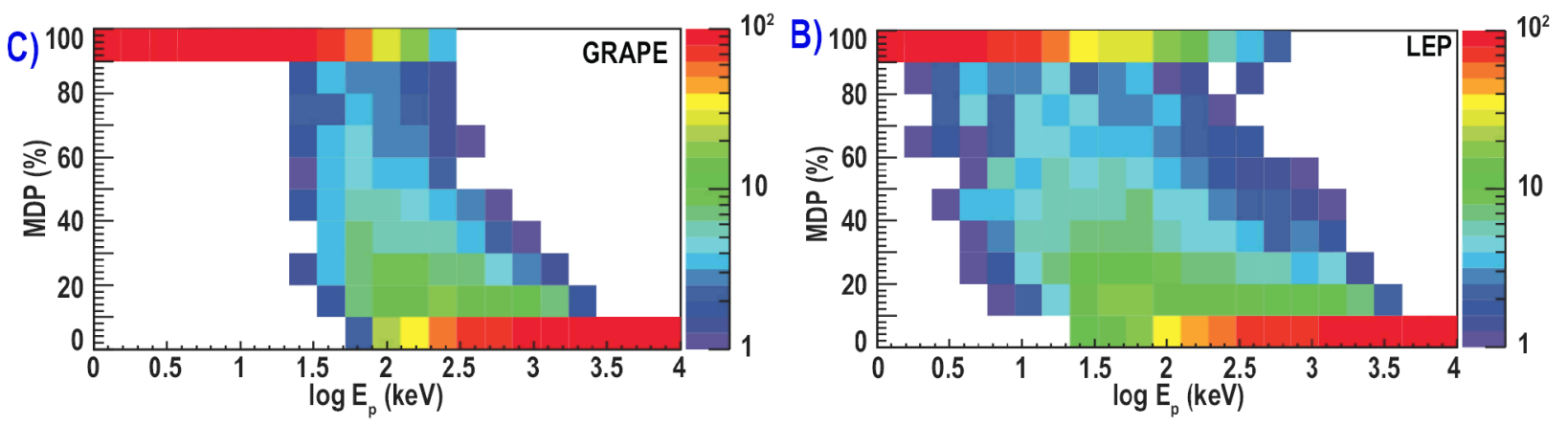

FIGURE 3. LEFT: GRAPE sensitivity to GRB polarization for a distribution of $E_{p}$ RIGHT: LEP sensitivity to GRB polarization for a distribution of $E_{p}$

Performance: $\mathrm{A}$ prototype detector has been characterized with polarized $\mathrm{X}$-rays at $6.4 \mathrm{keV}$ and $4.5 \mathrm{keV}$ and unpolarized $6 \mathrm{keV}$ X-rays in Ne:CO $2: \mathrm{CH}_{3} \mathrm{NO}_{2}$ at 500 and 600 Torr. A typical modulation curve is shown in Figure 2. The modulation factor at $6.4 \mathrm{keV}$ is $41 \%$ and the residual instrumental asymmetry measured with unpolarized X-rays is below $1 \%$. The instrument performance has been modeled over the full energy range. The LEP effective area for a source $30^{\circ}$ off-axis is shown in Figure 1, providing more than $200 \mathrm{~cm}^{2}$ at $3.5 \mathrm{keV}$.

GRB Sensitivity: To determine the sensitivity of the LEP, GRBs were selected from the HETE-2 catalog from the beginning of the mission to 13 September 2003 which were detected both in the wide-field X-ray monitor (WXM) and the French Gamma telescope (FREGATE). 45 bursts satisfied these criteria [16].

The MDP for each of the 45 bursts was calculated using a weighted modulation factor [11]. Accounting for the HETE-2 FoV ( 0.9 str) and the time period over which the catalog was obtained ( $~ 3$ years operational time including periods in the SAA), and scaling the distribution of bursts to the LEP field of view, the number of bursts for a given MDP was determined per year. LEP will detect $>8$ GRBs per year with a sensitivity of $10 \%$ and $>40 /$ year with a sensitivity of $25 \%$.

\section{POET Sensitivity}

44,100 GRBs were simulated, varying $E_{p}$ from $1 \mathrm{keV}$ to $10 \mathrm{MeV}$. The BATSE time-averaged Band function spectral parameter distribution was assumed for the low energy and high-energy photon indices and the BAT $T_{100}$ distribution for the duration. The time-averaged flux (2-400 keV) versus $E_{p}$ of the HETE-2 GRB sample [16] indicates that GRBs with $E_{p} \sim 10 \mathrm{keV}, 100 \mathrm{keV}$ and $1000 \mathrm{keV}$ tend to have a flux of $\sim 10^{-8} \mathrm{erg} \mathrm{cm}^{-2} \mathrm{~s}^{-1}, \sim 10^{-7} \mathrm{erg} \mathrm{cm}^{-2} \mathrm{~s}^{-1}$ and $\sim 10^{-6} \mathrm{erg} \mathrm{cm}^{-2} \mathrm{~s}^{-1}$, respectively. The LEP and GRAPE responses were calculated for the 12,500 simulated bursts that satisfied the HETE-2 relationship. The number of bursts detected with S/N $>5$ is $99 \%$ for LEP, $80 \%$ for GRAPE, $78 \%$ GRAPE and LEP). The number of bursts for which $E_{p}$ can be determined is $20 \%$ for $E_{p}<10 \mathrm{keV},>50 \%$ for $E_{p}$ $<20 \mathrm{keV}$ and $\sim 100 \%$ for $E_{p} \sim 0.2-1 \mathrm{MeV}$. The polarization sensitivity (i.e. MDP) to the burst sample is shown in Figure 3 where the z-axis is the percentage of bursts measured with a given $E_{p}$ and MDP.

\section{SUMMARY}

The capabilities presented here for GRAPE and LEP, show that the POET mission would significantly advance our understanding of key physical processes through high energy polarimetry and simultaneous broadband spectroscopy of Gamma-ray bursts.

\section{ACKNOWLEDGMENTS}

The authors would like to acknowledge the efforts of the entire POET team that proposed to the 2008 SMEX AO and the USRA proposal team that compiled much of the information presented in this paper. 


\section{REFERENCES}

1. D. Band et al., ApJ 413, 281 (1993)

2. J. K. Black et al. NIM A, 581, 755 (2007)

3. Y. Z. Fan, B. Zhang \& D. Proga, ApJ 635, L129 (2005)

4. N. Gehrels et al., Nature 437, 851(2005)

5. J. Granot, $A p J$ 596, L17 (2003)

6. J. E. Hill et al., Proc. SPIE 6686, 66860Y (2007)

7. D. Lazzati et al., MNRAS 347, L1 (2004)

8. M. Lyutikov, V. I. Pariev \& R. D. Blandford, ApJ 597, 998 (2003)

9. M. L. McConnell et al., Proc SPIE 5165, 334 (2004)

10. M. L. McConnell et al., NIMA, submitted

11. L. Pacciani et al., Proc SPIE 4843, 394 (2003)

12. B. Paczynski, ApJ 494, L45 (1998)

13. M. J. Rees \& P. Meszaros, $M$ NRAS 258, 41P (1992)

14. M. J. Rees \& P. Meszaros, ApJ 430, L93 (1994)

15. F. Ryde, ApJ 625, L95 (2005)

16. T. Sakamoto et al., ApJ 629, 311 (2005)

17. N. J. Shaviv and A. Dar, ApJ 447, 863 (1995)

18. S. J. Sturner et al., $A \& A$ 411, L81 (2003)

19. K. Toma et al., these proceedings

20. E. Waxman, Nature 423, 388 (2003)

21. B. Zhang \& P. Meszaros, Int. J. Mod. Phys. A 19, 2385 (2004) 